\title{
Real-World Treatment Patterns among Patients with Advanced Gastric Cancer in South Korea
}

\section{Gebra Cuyun Carter, $\mathrm{PhD}^{1}$ \\ Anna Kaltenboeck, $\mathrm{MA}^{2}$ \\ Jasmina Ivanova, $\mathrm{MA}^{2}$ \\ Astra M. Liepa, PharmD'1 \\ Alexandra San Roman, $\mathrm{BA}^{3}$ \\ Maria Koh, MSc² \\ Narayan Rajan, $\mathrm{MS}^{4}$ \\ Rebecca Cheng, MD \\ Howard G. Birnbaum, $\mathrm{PhD}^{3}$ \\ Jong Seok Kim, MD ${ }^{6}$ \\ Yung-Jue Bang, $\mathrm{MD}, \mathrm{PhD}^{7}$}

\author{
${ }^{1}$ Eli Lilly and Company, Indianapolis, IN, \\ ${ }^{2}$ Analysis Group, New York, NY, \\ ${ }^{3}$ Analysis Group, Boston, MA, USA, \\ ${ }^{4}$ Eli Lilly and Company, Sydney, Australia, \\ ${ }^{5}$ Eli Lilly and Company, Taipei, Taiwan, \\ ${ }^{6}$ Eli Lilly and Company, Seoul, \\ ${ }^{7}$ Department of Internal Medicine, \\ Seoul National University Hospital, \\ Seoul, Korea
}

\section{Purpose}

The purpose of this study was to understand patient treatment patterns, outcomes, and healthcare resource use in cases of metastatic and/or locally recurrent, unresectable gastric cancer (MGC) in South Korea.

\section{Materials and Methods}

Thirty physicians reviewed charts of eligible patients to collect de-identified data. Patients must have received platinum/fluoropyrimidine first-line therapy followed by second-line therapy or best supportive care, had no other primary cancer, and not participated in a clinical trial following MGC diagnosis. Data were summarized using descriptive statistics. KaplanMeier analysis was used to describe survival.

\section{Results}

Of 198 patients, $73.7 \%$ were male, $78.3 \%$ were diagnosed with MGC after age 55 (mean, 61.3 years), and $47.0 \%$ were current or former smokers. The majority of tumors were located in the antrum/pylorus (51.5\%). Metastatic sites most often occurred in the peritoneum (53.5\%), lymph nodes (47.5\%), and liver (38.9\%). At diagnosis, the mean Charlson comorbidity index was 0.4 (standard deviation, 0.6). The most common comorbidities were chronic gastritis $(22.7 \%)$ and cardiovascular disease (18.7\%). Most patients $(80.3 \%)$ received second-line treatment. Single-agent fluoropyrimidine was reported for $22.0 \%$ of patients, while $19.5 \%$ were treated with irinotecan and a fluoropyrimidine or platinum agent. The most common physician-reported symptoms during second-line treatment were nausea/vomiting (44.7\%) and pain (11.3\%), with antiemetics (44.7\%), analgesics (36.5\%), and nutritional support (11.3\%) most often used as supportive care. Two-thirds of inpatient hospitalizations were for chemotherapy infusion. Outpatient hospitalization (31.6\%) and visits to the oncologist (58.8\%) were common among second-line patients.

\section{Conclusion}

Most patients received second-line treatment, although regimens varied. Understanding MGC patient characteristics and treatment patterns in South Korea will help address unmet needs.
307 E. McCarty
IN 46285 , USA

Tel: 1-317-370-2937

Fax: 1-317-433-1304

E-mail: cuyun_carter_gebra@lilly.com

Received January 15, 2016

Accepted August 26, 2016

Published Online September 12, 2016

\section{Key words}

Treatment patterns, Stomach neoplasms, Republic of Korea, Resource use, Observational study

\section{Introduction}

Gastric cancer is the fourth most common cancer worldwide and the second most common cause of cancer-related deaths $[1,2]$. The incidence of gastric cancer is highest in East Asian countries and in some parts of South America, while its occurrence is lower in North America and Africa [1,3]. South Korea has the highest incidence rate of gastric cancer (age standardized, male vs. female: 64.2 and 26.7 per $100,000)$. Globally, a high proportion of patients are diagnosed with late-stage disease, and 5-year survival rates are $<25 \%$ for these patients. Gastric cancer is the second most commonly diagnosed cancer in South Korea, with male and 
female mortality rates of 37.1 and 15.0 per 100,000, respectively. Risk increases with advancing age, history of Helicobacter pylori infection and cigarette smoking [2,3].

There is high unmet need in gastric cancer as there are few approved agents, and treatment practices vary widely among countries [1,2], particularly in the second-line treatment setting. There is also limited information available on gastric cancer patient characteristics, healthcare resource use and treatment patterns $[1,2,4]$.

Therefore, this study was conducted to understand treatment patterns, patient outcomes, and healthcare resource use in South Korean patients with metastatic and/or locally recurrent, unresectable gastric cancer (MGC), including cancer of the stomach and gastroesophageal junction with adenocarcinoma histology.

\section{Materials and Methods}

This study is a retrospective analysis of de-identified patient-level data from medical charts collected via a physician-administered online chart review or face-to-face interviews with physicians who treated gastric cancer patients. Physicians selected at random from a panel of oncologists and referrals to the study by local contacts and elected to participate in the study provided de-identified patient-level data from a random sample of patient charts.

This study received Investigational Review Board (IRB) exemption from the Seoul National University Hospital and from the Western Institutional Review Board.

\section{Data collection}

Per protocol, each physician could provide information from up to 10 patient charts. Inclusion in the study was limited to adult patients ( $\geq 18$ years) diagnosed with MGC, including cancer of the stomach or gastroesophageal junction with adenocarcinoma histology, on or after January 1, 2009 (until data collection began in 2013). Patients could have been diagnosed with an earlier stage gastric cancer before January 1, 2009. Eligible patients had no other primary malignant tumors and completed platinum/fluoropyrimidine $(\mathrm{P} / \mathrm{F})$ first-line therapy (with or without other drugs; e.g., therapy with trastuzumab) after MGC diagnosis. Upon completion of first-line therapy, eligible patients either went on to (1) second-line therapy or (2) best supportive care (BSC) only. Patients were not eligible if they participated in any clinical trials after MGC diagnosis.

The chart abstraction instrument was designed to collect information on physician and patient characteristics, treat- ment patterns by line of therapy, patient outcomes, and health care resource use. Comorbidities were also collected to allow reporting of patients' Charlson comorbidity index (CCI). The CCI is a validated tool based on 17 comorbidities that is used to predict the risk of 1-year mortality [5].

\section{Statistical analysis}

Patient characteristics were compared between patients who received second-line therapy and those who received only BSC after first-line therapy. Chi-squared tests were used for comparisons of proportions, and Wilcoxon rank sum tests were used for comparisons of continuous variables. $p$-values of $<0.05$ were considered statistically significant. All data were evaluated descriptively with univariate analysis using SAS ver. 9.3 (SAS Institute Inc., Cary, NC).

Kaplan-Meier analysis was used to describe survival and disease progression. Survival time was calculated from (1) the date of MGC diagnosis to the date of death for all patients, and (2) from the date of initiation of second-line therapy to the date of death for the cohort that received second-line therapy. Patients surviving at the time of data collection were censored at the date of last contact. Patients who were reported to have died, but for whom no dates of death were available, were recorded as having died on the date of last contact.

Rates of disease progression defined as discontinuing any agent in that line of therapy due to disease progression were calculated for each line of therapy. Duration of each line of therapy was defined as the number of days from the first to the last administration of any agent in that line of therapy. Patients with ongoing second-line and third-line therapy were excluded from the calculations of mean duration of second-line and third-line therapy, respectively, but not from Kaplan-Meier analyses of survival and disease progression.

Healthcare resource utilization, including inpatient and outpatient office visits, was calculated for each line of therapy. Observations were excluded from the calculation of each resource use category if it was unknown in what line of therapy they occurred.

\section{Results}

\section{Physician characteristics}

Thirty physicians that were selected at random from a panel of oncologists and referrals to the study by local contacts and elected to participate in the study provided de-identified patient-level data from 198 patient charts. Most 
Table 1. Baseline patient and disease characteristics at diagnosis

\begin{tabular}{|c|c|}
\hline Variable & No. $(\%)$ \\
\hline MGC patient & $198(100)$ \\
\hline Age at MGC diagnosis, mean \pm SD & $61.3 \pm 9.8$ \\
\hline \multicolumn{2}{|l|}{ Age group at MGC diagnosis (yr) } \\
\hline $25-44$ & $12(6.1)$ \\
\hline $45-54$ & $31(15.7)$ \\
\hline $55-64$ & $73(36.9)$ \\
\hline$\geq 65$ & $82(41.4)$ \\
\hline Male & $146(73.7)$ \\
\hline Body mass index ${ }^{\mathrm{a})}$, mean $\pm \mathrm{SD}\left(\mathrm{kg} / \mathrm{m}^{2}\right)$ & $21.4 \pm 2.6$ \\
\hline \multicolumn{2}{|l|}{ Smoking history } \\
\hline Non-smoker & $83(41.9)$ \\
\hline Current smoker & $38(19.2)$ \\
\hline Former smoker & $55(27.8)$ \\
\hline Unknown & $22(11.1)$ \\
\hline \multicolumn{2}{|l|}{ Alcohol consumption } \\
\hline No alcohol use & $68(34.3)$ \\
\hline Light to moderate & $94(47.5)$ \\
\hline Heavy & $18(9.1)$ \\
\hline Unknown & $18(9.1)$ \\
\hline History of Helicobacter pylori infection & $8(4.0)$ \\
\hline Family history of gastric cancer & $13(6.6)$ \\
\hline Charlson comorbidity index $(\mathrm{CCI})^{\mathrm{b})}$, mean \pm SD & $0.4 \pm 0.6$ \\
\hline \multicolumn{2}{|l|}{ Common comorbidity } \\
\hline Chronic atrophic gastritis & $45(22.7)$ \\
\hline Cardiovascular disease & $37(18.7)$ \\
\hline Intestinal metaplasia & $28(14.1)$ \\
\hline Diabetes without chronic complications ${ }^{c)}$ & $24(12.1)$ \\
\hline Chronic obstructive pulmonary disease ${ }^{c)}$ & $16(8.1)$ \\
\hline Peptic ulcer disease ${ }^{c)}$ & $15(7.6)$ \\
\hline Stage IV at MGC diagnosis & $193(97.5)$ \\
\hline \multicolumn{2}{|l|}{ Disease classification at MGC diagnosis } \\
\hline Intestinal & $48(24.2)$ \\
\hline Diffuse & $72(36.4)$ \\
\hline Mixed & $13(6.6)$ \\
\hline Unknown & $65(32.8)$ \\
\hline \multicolumn{2}{|l|}{ Tumor location } \\
\hline Antrum and pylorus & $102(51.5)$ \\
\hline Fundus and corpus & $48(24.2)$ \\
\hline Gastric cardia & $25(12.6)$ \\
\hline Esophagogastric junction & $11(5.6)$ \\
\hline Whole stomach & $7(3.5)$ \\
\hline Other & $1(0.5)$ \\
\hline Unknown & $4(2)$ \\
\hline \multicolumn{2}{|l|}{ Metastatic site } \\
\hline Peritoneum & $106(53.5)$ \\
\hline Lymph nodes & $94(47.5)$ \\
\hline Liver & 77 (38.9) \\
\hline Bone & $22(11.1)$ \\
\hline Lung & $9(4.5)$ \\
\hline Other & $9(4.5)$ \\
\hline
\end{tabular}

Table 1. Continued

\begin{tabular}{lc} 
Variable & No. (\%) \\
Tested for HER2/neu gene expressiond) & $84(42.4)$ \\
HER2 positive & $8(9.5)$ \\
HER2 negative & $75(89.3)$ \\
HER2 status unknown & $1(1.2)$ \\
\hline
\end{tabular}

MGC, metastatic and/or locally recurrent, unresectable gastric cancer; SD, standard deviation; HER2, human epidermal growth factor receptor 2. ${ }^{\text {al }}$ Patients with weight less than $20 \mathrm{~kg}(\mathrm{n}=1)$ were assumed to have the population average weight of $58 \mathrm{~kg}$, ${ }^{\text {b }}$ The CCI was calculated excluding any malignancy (including leukemia and lymphoma) and metastatic solid tumor, ${ }^{\mathrm{c}}$ Comorbidities contributing to the CCI are marked, d'The proportions of patients with positive, negative, and unknown values for HER2/neu gene expression are only among the tested patients.

physicians $(27 / 30,90 \%)$ specialized in gastric oncology, and the average time in practice was 11.5 years (standard deviation [SD], 5.5 years). Nine of 30 physicians reported affiliation with one of the major cancer centers in South Korea based on a limited set of centers noted in the survey instruments. Data were collected from February 20, 2013, to April 29, 2013. No data identifying the physicians were collected, and only physicians had access to the medical charts during the abstraction process.

\section{Patient and disease characteristics}

Charts were abstracted for 198 MGC patients. At MGC diagnosis, patients were 61.3 years old (SD, 9.8) on average, and $73.7 \%$ were male. Most patients $(41.9 \%)$ had no history of smoking, while $19.2 \%$ and $27.8 \%$ were current or former smokers, respectively. Alcohol use was most frequently light to moderate $(47.5 \%)$, although $9.1 \%$ of patients had a history of heavy alcohol consumption. A history of $H$. pylori infection or a family history of gastric cancer was observed in $4.0 \%$ and $6.6 \%$ of patients, respectively (Table 1 ).

The most commonly reported comorbidities were chronic atrophic gastritis (22.7\%), cardiovascular disease (18.7\%), intestinal metaplasia (14.1\%), and diabetes without chronic complications (12.1\%). Excluding malignancy and metastatic solid tumor diagnoses, the mean CCI was 0.4 (SD, 0.6) (Table 1). 
Table 2. Patient status and treatment regimens by line of therapy

\begin{tabular}{|c|c|}
\hline Variable & No. $(\%)$ \\
\hline MGC patient & $198(100)$ \\
\hline $\begin{array}{l}\text { Patient who received first-line } \\
\text { chemotherapy treatment }\end{array}$ & $198(100)$ \\
\hline $\begin{array}{l}\text { Patient who received second-line } \\
\text { chemotherapy treatment }\end{array}$ & $159(80.3)$ \\
\hline $\begin{array}{l}\text { Patient who received third-line } \\
\text { chemotherapy treatment }\end{array}$ & $46(23.2)$ \\
\hline Patient who received BSC only after first-line & $39(19.7)$ \\
\hline \multicolumn{2}{|l|}{ ECOG PS score of first-line patientsa) } \\
\hline 0: Asymptomatic & $25(12.6)$ \\
\hline 1: Symptomatic but completely ambulatory & $151(76.3)$ \\
\hline 2: Symptomatic, $<50 \%$ in bed during the day & $19(9.6)$ \\
\hline 4: Bedbound & $2(1.0)$ \\
\hline Unknown & $1(0.5)$ \\
\hline \multicolumn{2}{|l|}{ First-line regimen $^{\text {b) }}$} \\
\hline Fluoropyrimidine+platinum (+/- leucovorin) & $120(60.6)$ \\
\hline Capecitabine+platinum & $29(14.6)$ \\
\hline Single-agent fluoropyrimidine (+/ - leucovorin) & $38(19.2)$ \\
\hline Reason for initiating second-line therapy & $159(100)$ \\
\hline Tumor progression & $152(95.6)$ \\
\hline Toxicity of first-line therapy & $7(4.4)$ \\
\hline ECOG PS score of second-line patients ${ }^{a}$ & $159(100)$ \\
\hline 0: Asymptomatic & $16(10.1)$ \\
\hline 1: Symptomatic but completely ambulatory & $105(66.0)$ \\
\hline 2: Symptomatic, $<50 \%$ in bed during the day & $35(22.0)$ \\
\hline Unknown & $3(1.9)$ \\
\hline Second-line regimen ${ }^{\text {b) }}$ & $159(100)$ \\
\hline Single agent fluoropyrimidine (+/- leucovorin) & $35(22.0)$ \\
\hline S-1 & $11(6.9)$ \\
\hline Capecitabine & $9(5.7)$ \\
\hline 5-FU & $7(4.4)$ \\
\hline $\begin{array}{l}\text { Irinotecan+platinum and/or } \\
\text { fluoropyrimidine }(+/- \text { leucovorin })\end{array}$ & $31(19.5)$ \\
\hline Irinotecan, 5-FU, leucovorin & $15(9.4)$ \\
\hline Irinotecan, 5-FU & $10(6.3)$ \\
\hline Fluoropyrimidine+ platinum agent & $21(13.2)$ \\
\hline \multicolumn{2}{|l|}{ (+/- leucovorin) } \\
\hline Capecitabine+platinum agent & $9(5.7)$ \\
\hline 5-FU+platinum agent & $7(4.4)$ \\
\hline S-1+platinum agent & $5(3.1)$ \\
\hline Single-agent taxane & $13(8.2)$ \\
\hline Docetaxel & $9(5.7)$ \\
\hline Otherc) & $59(37.1)$ \\
\hline ECOG score of third-line patient & $46(100)$ \\
\hline 0: Asymptomatic & $5(10.9)$ \\
\hline 1: Symptomatic but completely ambulatory & $25(54.3)$ \\
\hline 2: Symptomatic, $<50 \%$ in bed during the day & $16(34.8)$ \\
\hline Unknown & 0 \\
\hline
\end{tabular}

Table 2. Continued

\section{Variable}

No. $(\%)$

Third-line regimen

Single-agent fluoropyrimidine (+ / - leucovorin) 12 (26.1)

Fluoropyrimidine+platinum agent

(+/- leucovorin)

Single-agent taxane 9 (19.6)

Irinotecan+platinum and/or 7 (15.2)

fluoropyrimidine (+/- leucovorin)

Other

MGC, metastatic and/or locally recurrent, unresectable gastric cancer; ECOG PS, Eastern Cooperative Oncology Group performance status; 5-FU, 5-fluorouracil. ${ }^{a}$ Karnofsky scores were converted to ECOG PS scores (100 [ECOG PS 0], 80-90 [ECOG PS 1], 60-70 [ECOG PS 2], 40-50 [ECOG PS 3], and 10-30 [ECOG PS 4]), b) A patient could have received a maximum of four therapeutic agents, ${ }^{c}$ Other regimens included: irinotecan or oxaliplatin $(6.9 \%)$, leucovorin/ irinotecan $(6.3 \%)$, cisplatin $(5.7 \%)$, cisplatin/ docetaxel $(3.1 \%)$, and various other agents received by fewer than $2 \%$ of patients.

\section{Disease and tumor characteristics}

Upon initial gastric cancer diagnosis, $76.8 \%$ of patients had stage IV disease according to the American Joint Committee on Cancer TNM (tumor size, lymph nodes affected, metastases) system [6]. At MGC diagnosis, 97.5\% of patients had stage IV disease, with diffuse histology (by the Laurén system [7]) being the most frequently reported type $(33.3 \%)$. There were no patients with stage III disease, and the staging for the remaining $2.5 \%$ of patients was unknown/ other. Histology information was missing for $32.8 \%$ of patients. The antrum and pylorus were the primary tumor locations in the majority of patients $(51.5 \%)$. The most frequently reported metastatic sites were the peritoneum (53.5\%), lymph nodes $(47.5 \%)$, and liver $(38.9 \%)$ (Table 1$)$. On average, MGC diagnosis occurred 4.5 months (SD, 14.3) after initial gastric cancer diagnosis.

Only 84 of the 198 patients were tested for HER2 positivity, of which $9.5 \%$ had positive status (Table 1). Variability in HER2 positivity rates of testing over a 5 -year period including 2009 to 2013 ranged from $0 \%$ in 2009 to $52.0 \%$ in 2012, with an overall rate of testing of $42.4 \%$ for patients in this sample. A higher percentage of patients were tested for HER2 status at major cancer centers $(68.0 \%)$ than patients treated in other centers $(30.0 \%)$. 


\section{Treatment patterns}

\section{1) First-line therapy}

By design, all patients in the study were required to have first-line therapy for MGC. At therapy initiation, most patients $(76.3 \%)$ were symptomatic but completely ambulatory (Eastern Cooperative Oncology Group performance status [ECOG PS], 1), while some remained asymptomatic (ECOG PS, $0 ; 12.6 \%$ ).

In this sample of 198 patient charts, the most frequent first- line regimen type was a fluoropyrimidine with a platinum agent (+/- leucovorin) $(60.6 \%)$, which consisted primarily of 5 -fluorouracil with a platinum agent $(40.9 \%)$, capecitabine with a platinum agent $(14.6 \%)$, or S-1 with a platinum agent (5.1\%). Single-agent fluoropyrimidine (+/ - leucovorin) was prescribed for $19.2 \%$ of patients, and in fewer patients, irinotecan plus platinum and / or fluoropyrimidine (+ / - leucovorin) $(4.5 \%)$ was prescribed (Table 2). For $88.9 \%$ of patients, physicians reported selecting first-line treatment based on national guidelines. For $22.7 \%$ of patients, physician experience was a factor in selection of the first-line treatment

Table 3. Patient demographic characteristics stratified by BSC or second-line therapy after first-line therapy

\begin{tabular}{|c|c|c|c|}
\hline Variable & $\begin{array}{l}\text { First-line followed } \\
\text { by BSC ( } n=39)\end{array}$ & $\begin{array}{l}\text { First-line followed } \\
\text { by second-line }(n=159)\end{array}$ & p-value $e^{a}$ \\
\hline \multicolumn{4}{|l|}{ Age at MGC diagnosis (yr) } \\
\hline Years & $64.5 \pm 9.3$ & $60.5 \pm 9.8$ & $0.012^{*}$ \\
\hline Median (Q1-Q3) & $66(60-71)$ & $62(55-67)$ & \\
\hline \multicolumn{4}{|l|}{ Distribution (yr) } \\
\hline $25-34$ & $1(2.6)$ & $3(1.9)$ & $>0.990$ \\
\hline $35-44$ & 0 & $8(5.0)$ & 0.360 \\
\hline $45-54$ & $5(12.8)$ & $26(16.4)$ & 0.587 \\
\hline $55-64$ & $11(28.2)$ & $62(39.0)$ & 0.211 \\
\hline$\geq 65$ & $22(56.4)$ & $60(37.7)$ & $0.034^{*}$ \\
\hline Male & $31(79.5)$ & $115(72.3)$ & 0.363 \\
\hline \multicolumn{4}{|l|}{ Ethnicity } \\
\hline East Asian & $39(100)$ & $159(100)$ & $>0.990$ \\
\hline BMI $\left(\mathrm{kg} / \mathrm{m}^{2}\right)^{\mathrm{b})}$ & $21.8 \pm 2.8$ & $21.3 \pm 2.5$ & 0.121 \\
\hline \multicolumn{4}{|l|}{ Smoking history } \\
\hline Non-smoker & $18(46.2)$ & $65(40.9)$ & 0.550 \\
\hline Current smoker & $9(23.1)$ & $29(18.2)$ & 0.492 \\
\hline Former smoker & $11(28.2)$ & $44(27.7)$ & 0.947 \\
\hline Unknown & $1(2.6)$ & $21(13.2)$ & 0.084 \\
\hline \multicolumn{4}{|l|}{ Alcohol consumption } \\
\hline No alcohol use & $11(28.2)$ & $57(35.8)$ & 0.368 \\
\hline Light to moderate & $19(48.7)$ & $75(47.2)$ & 0.862 \\
\hline Heavy & $6(15.4)$ & $12(7.5)$ & 0.131 \\
\hline Unknown & $3(7.7)$ & $15(9.4)$ & $>0.990$ \\
\hline \multicolumn{4}{|l|}{ Performance status (ECOG score) } \\
\hline 0: Asymptomatic & $1(2.6)$ & $24(15.1)$ & $0.033^{*}$ \\
\hline 1: Symptomatic but completely ambulatory & $31(79.5)$ & $120(75.5)$ & 0.597 \\
\hline 2: Symptomatic, $<50 \%$ in bed during the day & $5(12.8)$ & $14(8.8)$ & 0.542 \\
\hline 3: Symptomatic, $>50 \%$ in bed, but not bedbound & 0 & 0 & - \\
\hline 4: Bedbound & $2(5.1)$ & 0 & $0.038^{*}$ \\
\hline Unknown & 0 & $1(0.6)$ & $>0.990$ \\
\hline
\end{tabular}

Values are presented as mean \pm standard deviation or number $(\%)$ unless otherwise indicated. BSC, best supportive care; MGC, metastatic and / or locally recurrent, unresectable gastric cancer; BMI, body mass index; ECOG, Eastern Cooperative Oncology

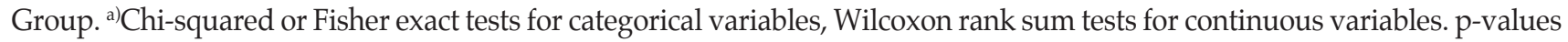
of $<0.05$ are indicated by an asterisk $\left({ }^{*}\right)$, b) Patients weighing less than $20 \mathrm{~kg}(\mathrm{n}=1)$ were assumed to have a population average weight of $58 \mathrm{~kg}$, c)Performance status was assessed at the beginning of first-line treatment. 


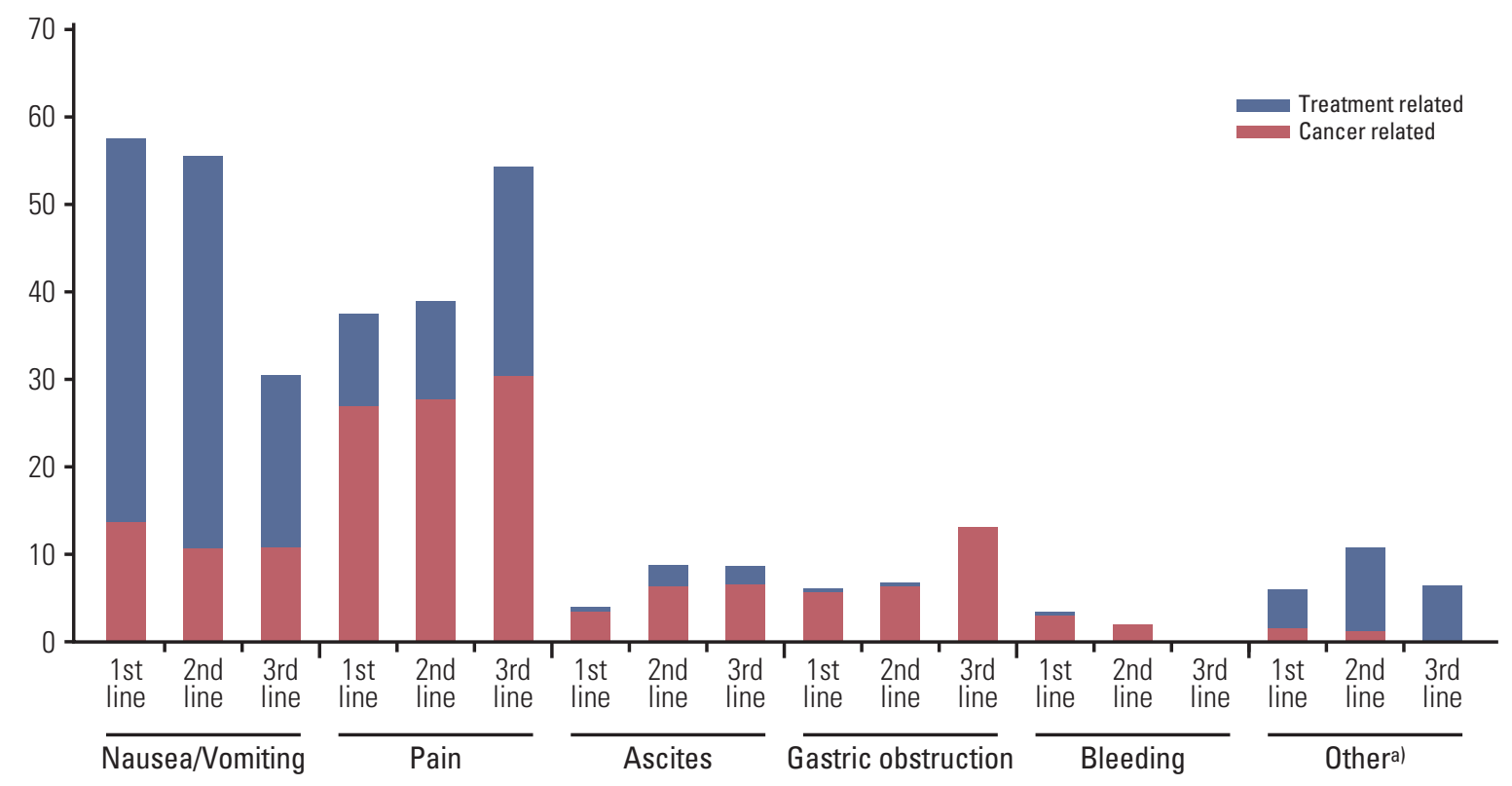

Fig. 1. Physician-reported symptoms (\% of patients) by line of therapy. ${ }^{a}$ Other symptoms or complications included bruises, diarrhea, neuralgia, mucositis, weakness, fatigue, hand numbness, hand-foot reaction, dermatitis, varicella, weakness of whole body, and leukopenia.

regimen.

First-line treatment lasted a median of 84 days (interquartile range [IQR], 49 to 155 days). Response to treatment included stable disease $(52.0 \%)$, partial response $(25.3 \%)$, disease progression $(18.2 \%)$, complete response $(3.0 \%)$, and unknown $(1.5 \%)$. In $75.8 \%$ of patients, first-line therapy was discontinued due to disease progression, while it was discontinued in $12.6 \%$ of patients because of an adverse event (AE) or toxicity. Other reasons for discontinuation included patient preference, completion of protocol, lack of benefit, and unknown.

\section{2) Second-line therapy and BSC}

In this study, 39 patients $(19.7 \%)$ received BSC only following first-line therapy, while $159(80.3 \%)$ received second-line therapy.

Second-line therapy was initiated due to tumor progression in $95.6 \%$ of patients. When qualitatively compared with first-line regimens, second-line regimens were more heterogeneous. Physicians reported treating $22.0 \%$ of patients with a single agent fluoropyrimidine (+ / - leucovorin), $19.5 \%$ with irinotecan with a platinum agent and/or fluoropyrimidine (+/- leucovorin), $13.2 \%$ with fluoropyrimidine with a platinum agent (+/- leucovorin), and $8.2 \%$ with single-agent taxane. Other types of regimens were used in $37.1 \%$ of patients
(Table 2). For $80.5 \%$ of patients, physicians reported selecting second-line therapy based on national guidelines, while in $34.6 \%$, selection was guided at least in part by experience.

Second-line treatment lasted a median of 64 days (IQR, 37 to 105 days). The most frequent best response to therapy was stable disease $(45.9 \%)$. Partial response was observed in $15.1 \%$ of patients, complete response in $1.3 \%$, progression occurred in $22.6 \%$, and response was unknown for $15.1 \%$. Second-line therapy was discontinued because of disease progression in $61.6 \%$ of patients, patient refusal in $17.6 \%$, AEs or toxicity in $11.3 \%$, lack of benefit in $2.5 \%$, other in $1.3 \%$, end of protocol in $0.6 \%$, and for unknown reasons in $13.2 \%$ of patients. When we stratified patients between those who received BSC and those who received second-line therapy, we found differences only in age and ECOG PS. Patients who received second-line therapy after first-line treatment were younger and more likely to be asymptomatic (Table 3).

\section{3) Third-line therapy}

Third-line therapy was administered in $23.2 \%$ of patients (46 out of 198). Due to the small number of patients who received third-line therapy, treatment patterns were not evaluated in detail. 
Table 4. Patient supportive care and hospitalization stratified by line of therapy

\begin{tabular}{|c|c|c|c|c|}
\hline Variable & $\begin{array}{l}\text { First-line } \\
(\mathbf{n}=198)\end{array}$ & $\begin{array}{l}\text { Second-line } \\
\qquad(n=159)\end{array}$ & $\begin{array}{c}\text { BSC, no } \\
\left.\text { second-line }^{a}\right)(n=39)\end{array}$ & $\begin{array}{l}\text { Third-line } \\
(\mathrm{n}=46)\end{array}$ \\
\hline \multicolumn{5}{|l|}{ Supportive care } \\
\hline Antiemetics & $103(52.0)$ & $71(44.7)$ & 7 (17.9) & $21(45.7)$ \\
\hline Analgesics & $74(37.4)$ & $58(36.5)$ & $9(23.1)$ & $13(28.3)$ \\
\hline Granulocyte-colony stimulating factors & $11(5.6)$ & $13(8.2)$ & 0 & $3(6.5)$ \\
\hline Diuretics & $9(4.5)$ & $6(3.8)$ & $1(2.6)$ & $1(2.2)$ \\
\hline Antidepressants & $6(3.0)$ & $4(2.5)$ & $1(2.6)$ & 0 \\
\hline Erythropoiesis stimulating agents & $4(2.0)$ & $2(1.3)$ & 0 & 0 \\
\hline GM-colony stimulating factors & $2(1.0)$ & 0 & 0 & 0 \\
\hline Narcotics & 0 & $1(0.6)$ & 0 & 0 \\
\hline Nutritional support & $27(13.6)$ & $18(11.3)$ & $4(10.3)$ & $4(8.7)$ \\
\hline \multicolumn{5}{|l|}{ Inpatient hospitalization } \\
\hline At least one stay & $71(35.9)$ & $48(30.2)$ & $13(33.3)$ & $11(23.9)$ \\
\hline No. of visits / patient & $3.5 \pm 3.4$ & $2.1 \pm 1.4$ & $2.5 \pm 2.5$ & $3.7 \pm 2.0$ \\
\hline Length of stay/hospitalization (day) & $8.2 \pm 9.4$ & $9.1 \pm 11.3$ & $14.3 \pm 15.6$ & $10.2 \pm 12.5$ \\
\hline \multicolumn{5}{|l|}{ Main reasons for visit } \\
\hline Chemotherapy infusion & $182(73.4)$ & $68(68.7)$ & $17(53.1)^{b)}$ & $23(56.1)$ \\
\hline Disease symptom management & $38(15.3)$ & $21(21.2)$ & $9(28.1)$ & $11(26.8)$ \\
\hline Adverse events/toxicity & $19(7.7)$ & $6(6.1)$ & $2(6.3)$ & $4(9.8)$ \\
\hline Pain management & $2(0.8)$ & $2(2.0)$ & $3(9.4)$ & $3(7.3)$ \\
\hline Gastric cancer-related surgery & $6(2.4)$ & 0 & $1(3.1)$ & 0 \\
\hline Regular monitoring & 0 & $2(2.0)$ & 0 & 0 \\
\hline $\begin{array}{l}\text { Outpatient hospitalization } \\
\text { (patients with information available) }\end{array}$ & 92 & 79 & 13 & 21 \\
\hline At least one visit & $59(64.1)$ & $25(31.6)$ & $4(30.8)$ & $7(33.3)$ \\
\hline Mean visits/patient & $3.2 \pm 4.3$ & $2.5 \pm 3.3$ & $1 \pm 0.0$ & $2.7 \pm 2.5$ \\
\hline Hospice unit (patients with information available) & 34 & 29 & 5 & 10 \\
\hline At least one stay & $1(2.9)$ & 0 & $1(20)$ & $2(20.0)$ \\
\hline Oncologist clinic (patients with information available) & 127 & 102 & 25 & 28 \\
\hline At least one visit & $86(67.7)$ & $60(58.8)$ & $9(36)$ & $10(35.7)$ \\
\hline No. of visits / patient & $3.9 \pm 3.3$ & $3.0 \pm 3.6$ & $5.1 \pm 5.2$ & $2.8 \pm 1.9$ \\
\hline
\end{tabular}

Values are presented as number (\%) or mean \pm standard deviation unless otherwise indicated. BSC, best supportive care; GM, granulocyte-macrophage. ${ }^{a} \mathrm{BSC}$ was defined as having received first-line therapy but not continuing to second-line therapy, b)Hospitalizations were classified as occurring in a particular line of therapy if there was overlap in the dates of hospitalization and the line of therapy. Hospitalizations for chemotherapy infusion that overlapped between first-line therapy and BSC were counted toward both lines of treatment.

\section{Physician-reported patient symptoms, supportive care, and healthcare resource use}

For each line of therapy, physicians reported symptoms in patients related to either cancer or cancer treatments. The most common cancer-related symptoms or complications reported were pain in $26.3 \%, 27.7 \%$, and $30.4 \%$, and nausea/vomiting in $13.6 \%, 10.7 \%$, and $10.9 \%$ of patients during first-, second-, and third-line therapy, respectively. Gastric obstruction was recorded in $5.6 \%, 6.3 \%$, and $13.0 \%$, ascites in $3.5 \%, 6.3 \%$, and $6.5 \%$, and bleeding in $3.0 \%, 1.9 \%$, and $0 \%$ of patients undergoing first-, second-, and third-line therapy, respectively (Fig. 1).

Symptoms related to cancer treatment were similar to those recorded for cancer. Nausea/vomiting was reported in $43.9 \%, 44.7 \%$, and $19.6 \%$, pain in $10.6 \%, 11.3 \%$, and $23.9 \%$, and ascites in $0.5 \%, 2.5 \%$, and $2.2 \%$ of patients undergoing first-, second-, and third-line therapies, respectively. Treatment-related symptoms were similar during second-line treatment, with nausea/vomiting (44.7\%) and pain (11.3\%) being the most common. Fewer than $1 \%$ of patients experienced gastric obstruction or bleeding during any line of treat- 
ment.

Supportive care needs were common during first-, second-, and third-line therapies as well as BSC, and most frequently consisted of antiemetics $(52.0 \%, 44.7 \%$ and $45.7 \%$, $17.9 \%)$, analgesics $(37.4 \%, 36.5 \%$ and $28.3 \%, 23.1 \%)$, and granulocyte colony-stimulating factors $(5.6 \%, 8.2 \%$ and $6.5 \%, 0 \%)$. Nutritional support was provided to $13.6 \%, 11.3 \%$ and $8.7 \%$ of patients undergoing first-, second-, and third-line treatment and $4 \%$ of patients during BSC (Table 4 ). The most commonly performed procedure was endoscopy, which was conducted in $18.2 \%, 7.5 \%$, and $4.3 \%$ of patients during first-, second-, and third-line treatment, respectively, and in 10.3\% of patients in BSC.

Inpatient hospitalizations were reported in $35.9 \%$ of patients during first-line treatment, $33.3 \%$ of patients receiving BSC only, and $30.2 \%$ of patients during second-line treatment. Chemotherapy infusions and disease symptom management were the most commonly cited reasons for inpatient hospitalizations across all groups (Table 4).

Outpatient hospitalizations, which were most frequently for disease symptom management or $\mathrm{AE} /$ toxicity, occurred in $64.1 \%$ of patients during first-line treatment, $31.6 \%$ of patients in second-line treatment, $33.3 \%$ of patients during third-line treatment, and $30.8 \%$ of patients receiving BSC. Visits to oncology clinics, which were most commonly associated with pain management, were reported in $67.7 \%$, $58.8 \%, 35.7 \%$, and $36.0 \%$ of patients receiving first-, second-, and third-line treatment, and BSC, respectively (Table 4).

\section{Survival and disease progression}

Overall, the median survival time was 26.8 months (IQR, 9.9 to 41.6 months) from MGC diagnosis with $72.7 \%$ of patients censored. Patients who received second-line therapy had a median survival of 28.1 months (IQR, 10.5 to 36.6 months) with $74.2 \%$ of patients censored. Patients who received BSC only following first-line therapy had a median survival of 20.1 months (IQR, 8.5 to 41.6 months), with $66.7 \%$ of patients censored.

Among patients who received second-line therapy, median survival from initiation of second-line treatment was 13.0 months (IQR, 4.5 to 24.7 months) with $59.6 \%$ of patients censored.

\section{Discussion}

Our observational study supplements the information currently available regarding treatment of MGC in South Korea. We found variations in treatment patterns in both first- and second-line treatment regimens of patients with MGC. These findings are consistent with the results reported for a REGATE study in which $96 \%$ of patients received adjuvant chemotherapy consisting of varying agents, combinations and routes of administration [4]. Recently published guidelines for the diagnosis and treatment of gastric cancer in Korea [8] recommend fluoropyrimidines, platinums, taxanes, irinotecan, and anthracyclines as first-line chemotherapy and state no standard for second-line has been established, while these guidelines were published prior to recent approvals the second line [9-11]. In the present study, the selected patients were required to have $\mathrm{P} / \mathrm{F}$ first-line therapy (with or without other drugs; i.e., therapy with trastuzumab) after MGC diagnosis, but these criteria were not strictly adhered to and impacted the treatment regimens observed during first-line therapy. Following first-line treatment, $80.3 \%$ of the studied patients received second-line treatment, while $19.7 \%$ of patients received BSC. The large proportion of patients receiving second-line treatment rather than BSC only may have been due to recent reports of the clinical benefits of second-line chemotherapy to overall survival and quality of life [12-14]. In the REAL-2 study, which was a randomized, controlled clinical trial, only $14 \%$ of patients received secondline treatment [15]. More recently, in the AVAGAST trial, 66\% of Asian patients received second-line treatment [16]. Patients in our study were required to have had either second-line therapy or BSC after first-line therapy. These criteria may impact survival estimates following MGC diagnosis. Patients in our study who received second-line treatment were younger and had higher ECOG PS than those who received BSC. Performance status was reported to be a predictor of response to chemotherapy [17]. A recent metaanalysis suggests that patients with PS 0 have better survival after chemotherapy than those with PS 1 [18]. Among patients who received second-line therapy in the present study, $61.6 \%$ discontinued treatment because of disease progression and 46 patients $(23.2 \%)$ received third-line treatment, highlighting the need for more clinical trials in advanced gastric cancer.

Overall, $42 \%$ of patients in this sample were tested for HER2 positivity, with a larger proportion of patients tested annually during the more recent years of the study. Of those tested, 9.5\% tested positive for HER2. This rate of HER2 expression is consistent with the $6 \%-35 \%$ rate reported for gastric cancers $[12,19]$. HER2 testing is recommended in Korean gastric cancer treatment guidelines and in the ESMO and National Comprehensive Cancer Network guidelines $[8,20,21]$. Current guidelines recommend the addition of trastuzumab to chemotherapy in HER2+ patients [22].

Physician recommendations for MGC first-line therapy were most influenced by national guidelines and publications in top clinical journals. While the majority of first-line 
regimens administered to patients in this study consisted of a platinum agent and fluoropyrimidine as per eligibility criteria, second-line therapies varied widely, and include regimens that consisted of various single-agent fluoropyrimidines, doublet and triplet regimens, and single-agent taxanes. In a large, international prospective study of gastric cancer treatment (REGATE I), the use of a taxane such as paclitaxel, docetaxel, or irinotecan was reportedly the suggested second-line treatment for gastric cancer in Asia, though fewer than $10 \%$ of patients in the present sample were prescribed a taxane [1]. Other recent studies have reported a survival advantage associated with treatment with a second-line taxane such as docetaxel, or with irinotecan for cancers refractory to fluoropyrimidine and platinum treatment when compared to BSC $[13,14]$. In addition, no single agent option has been shown to be better than another in the second-line setting $[18,23]$. In our data, there appeared to be a trend in the increased use of taxanes as second-line therapy in 2012 relative to earlier years of the study (2009-2011); however, taxanes were prescribed to less than $20 \%$ of patients.

Best response to therapy and reasons for discontinuation of first- and second-line therapies revealed a significant unmet need in the treatment of MGC. Most patients achieved at most partial response or stable disease while on first-line therapy, and most discontinued this treatment due to disease progression. Similarly, the reason for initiating second-line therapy was tumor progression in $96 \%$ of patients. Responses to second-line therapy were less favorable than those to the first-line. In addition to disease progression, a major reason for discontinuing second-line therapy was patient refusal.

Healthcare resource use was driven both by chemotherapy administration and symptom management. The most commonly reported cancer treatment-related symptoms experienced by patients were nausea/vomiting and pain, and the most commonly used supportive care agents were antiemetics and analgesics. In addition to inpatient hospitalization visits for chemotherapy administration, patients were often seen in outpatient hospitals and oncology clinics for management of disease symptoms, AEs, toxicities, and pain.

It should be noted that this study is subject to the limitations of physician-administered chart abstractions. The completeness and accuracy of collected patient-level information depended on the accuracy of the physician recording the medical history information and treatment information, as well as the availability of a complete medical history in patient charts. Automated quality control checks for survey questions helped minimize possible inconsistencies in the recording of information. Patient-reported information documented in medical records and abstracted in this study may have been subject to self-report bias, including histories of smoking and alcohol use. Moreover, physicians may not have had full access to records documenting medical care administered to the patients over the course of MGC treatment, or to medical history prior to MGC diagnosis. Because a physician agreement was needed to participate, selection bias may play a role and treatment pattern information may not be representative of the treatment practice of all physicians or the treatments for all MGC patients in South Korea. It is important to interpret these results in light of the fact that the timeframe of this study was prior to more recent evidence supporting new therapies to guide practice in this space [9-11]. In addition, more than $70 \%$ of the study population was censored for survival, which is relatively high and may limit interpretation of the survival data.

\section{Conclusion}

The present study documents the high disease burden of gastric cancer and the significant unmet need that exists, particularly in the second-line setting. This study may help inform clinical practice and future research to ultimately improve patient outcomes.

\section{Conflicts of Interest}

This study was supported by Eli Lilly and Company. The following authors are employees of Eli Lilly and Company and may own company stock: G.C.C., A.M.L., N.R., R.C., and J.S.K.

\section{Acknowledgments}

The authors wish to thank the physicians who participated in the data collection for this study. 


\section{References}

1. Bang YJ, Yalcin S, Roth A, Hitier S, Ter-Ovanesov M, Wu CW, et al. Registry of gastric cancer treatment evaluation (REGATE): I baseline disease characteristics. Asia Pac J Clin Oncol. 2014;10:38-52.

2. Ferlay J, Soerjomataram I, Dikshit R, Eser S, Mathers C, Rebelo $\mathrm{M}$, et al. Cancer incidence and mortality worldwide: sources, methods and major patterns in GLOBOCAN 2012. Int J Cancer. 2015;136:E359-86.

3. Jung KW, Won YJ, Kong HJ, Oh CM, Cho H, Lee DH, et al. Cancer statistics in Korea: incidence, mortality, survival, and prevalence in 2012. Cancer Res Treat. 2015;47:127-41.

4. Ter-Ovanesov M, Yalcin S, Zalcberg J, Hitier S, Bang YJ, Wu $\mathrm{CW}$, et al. Registry of gastric cancer treatment evaluation (REGATE): II treatment practice. Asia Pac J Clin Oncol. 2013;9:373-80.

5. Charlson ME, Pompei P, Ales KL, MacKenzie CR. A new method of classifying prognostic comorbidity in longitudinal studies: development and validation. J Chronic Dis. 1987;40: 373-83.

6. Sobin LH, Fleming ID. TNM Classification of Malignant Tumors, fifth edition (1997). Union Internationale Contre le Cancer and the American Joint Committee on Cancer. Cancer. 1997;80:1803-4.

7. Hu B, El Hajj N, Sittler S, Lammert N, Barnes R, Meloni-Ehrig A. Gastric cancer: classification, histology and application of molecular pathology. J Gastrointest Oncol. 2012;3:251-61.

8. Lee JH, Kim JG, Jung HK, Kim JH, Jeong WK, Jeon TJ, et al. Clinical practice guidelines for gastric cancer in Korea: an evidence-based approach. J Gastric Cancer. 2014;14:87-104.

9. Wilke H, Muro K, Van Cutsem E, Oh SC, Bodoky G, Shimada $\mathrm{Y}$, et al. Ramucirumab plus paclitaxel versus placebo plus paclitaxel in patients with previously treated advanced gastric or gastro-oesophageal junction adenocarcinoma (RAINBOW): a double-blind, randomised phase 3 trial. Lancet Oncol. 2014;15:1224-35.

10. Fuchs CS, Tomasek J, Yong CJ, Dumitru F, Passalacqua R, Goswami C, et al. Ramucirumab monotherapy for previously treated advanced gastric or gastro-oesophageal junction adenocarcinoma (REGARD): an international, randomised, multicentre, placebo-controlled, phase 3 trial. Lancet. 2014;383: 31-9.

11. Ford HE, Marshall A, Bridgewater JA, Janowitz T, Coxon FY, Wadsley J, et al. Docetaxel versus active symptom control for refractory oesophagogastric adenocarcinoma (COUGAR-02): an open-label, phase 3 randomised controlled trial. Lancet Oncol. 2014;15:78-86.

12. Bang YJ, Van Cutsem E, Feyereislova A, Chung HC, Shen L, Sawaki A, et al. Trastuzumab in combination with chemotherapy versus chemotherapy alone for treatment of HER2-positive advanced gastric or gastro-oesophageal junction cancer
(ToGA): a phase 3, open-label, randomised controlled trial. Lancet. 2010;376:687-97.

13. Kang JH, Lee SI, Lim DH, Park KW, Oh SY, Kwon HC, et al. Salvage chemotherapy for pretreated gastric cancer: a randomized phase III trial comparing chemotherapy plus best supportive care with best supportive care alone. J Clin Oncol. 2012;30:1513-8.

14. Thuss-Patience PC, Kretzschmar A, Bichev D, Deist T, Hinke A, Breithaupt K, et al. Survival advantage for irinotecan versus best supportive care as second-line chemotherapy in gastric cancer: a randomised phase III study of the Arbeitsgemeinschaft Internistische Onkologie (AIO). Eur J Cancer. 2011;47: 2306-14.

15. Cunningham D, Starling N, Rao S, Iveson T, Nicolson M, Coxon $\mathrm{F}$, et al. Capecitabine and oxaliplatin for advanced esophagogastric cancer. N Engl J Med. 2008;358:36-46.

16. Ohtsu A, Shah MA, Van Cutsem E, Rha SY, Sawaki A, Park $\mathrm{SR}$, et al. Bevacizumab in combination with chemotherapy as first-line therapy in advanced gastric cancer: a randomized, double-blind, placebo-controlled phase III study. J Clin Oncol. 2011;29:3968-76.

17. Wilson D, Hiller L, Geh JI. Review of second-line chemotherapy for advanced gastric adenocarcinoma. Clin Oncol (R Coll Radiol). 2005;17:81-90.

18. Iacovelli R, Pietrantonio F, Farcomeni A, Maggi C, Palazzo A, Ricchini F, et al. Chemotherapy or targeted therapy as secondline treatment of advanced gastric cancer: a systematic review and meta-analysis of published studies. PLoS One. 2014;9: e108940.

19. Bang YJ. Advances in the management of HER2-positive advanced gastric and gastroesophageal junction cancer. J Clin Gastroenterol. 2012;46:637-48.

20. Ajani JA, Barthel JS, Bekaii-Saab T, Bentrem DJ, D'Amico TA, Das P, et al. Gastric cancer. J Natl Compr Canc Netw. 2010;8:378-409.

21. National Comprehensive Cancer Network. Gastric cancer (version 3.2015) [Internet]. Fort Washington, PA: National Comprehensive Cancer Network; 2015 [cited 2015 Jun 3]. Available from: http://www.nccn.org/professionals/physician_gls/pdf/gastric.pdf.

22. Elimova E, Shiozaki H, Wadhwa R, Sudo K, Chen Q, Estrella JS, et al. Medical management of gastric cancer: a 2014 update. World J Gastroenterol. 2014;20:13637-47.

23. Hironaka S, Ueda S, Yasui H, Nishina T, Tsuda M, Tsumura $\mathrm{T}$, et al. Randomized, open-label, phase III study comparing irinotecan with paclitaxel in patients with advanced gastric cancer without severe peritoneal metastasis after failure of prior combination chemotherapy using fluoropyrimidine plus platinum: WJOG 4007 trial. J Clin Oncol. 2013;31:4438-44. 